\title{
Sensitivity of the ANTARES neutrino telescope for secluded dark matter searches
}

\author{
The ANTARES Collaboration ${ }^{* *}$ \\ \$ http://antares.in2p3.fr/Collaboration/index2.html \\ E-mail: sara.gozzinieific.uv.es
}

\section{Filippo Sala}

Deutsches Elektonen-Synchrotron DESY

E-mail: filippo.sala@desy.de

\begin{abstract}
Dark matter scenarios starring WIMPs (weakly interacting massive particles) at the GeV-TeV scale have been intensively investigated, among others by neutrino telescopes with indirect searches. So far no WIMP candidate particle has been observed. At the same time, collider experiments are pushing the scale of the needed new physics beyond the $\mathrm{TeV}$ range, and such heavy sectors have long been known to provide dark matter candidates in the same mass range. This situation strongly motivates the exploration of DM scenarios with masses at and above 10$100 \mathrm{TeV}$. 'Secluded' frameworks then emerge as an ideal target for searches of the annihilation products of such heavy Dark Matter: they allow to naturally evade the unitarity bound on the dark matter mass, and at the same time to reliably compute the annihilation spectra of relevance for experiments. These perspectives open a new territory accessible to neutrino telescopes such as ANTARES and KM3NeT. Promising dark matter sources such as the Galactic Centre are in good visibility for these telescopes, that perform an unbinned-likelihood analysis in search for highenergy neutrinos produced in a dark matter pair-annihilation process. Current limits on WIMP pair annihilation cross section encourage the extension at higher energy, where the highest sensitivity is reached. The current status of secluded dark matter searches with ANTARES and the sensitivities achieved are presented.
\end{abstract}

Corresponding authors: Sara Rebecca Gozzini ${ }^{\dagger 1}$, Cristina Lagunas ${ }^{1}$, Filippo Sala $^{2}$, Juan de Dios Zornoza Gómez ${ }^{1}$

${ }^{1}$ IFIC (Univ. of Valencia - CSIC)

${ }^{2}$ Deutsches Elektonen-Synchrotron DESY

36th International Cosmic Ray Conference -ICRC2019-

July 24th - August 1st, 2019

Madison, WI, U.S.A.

\footnotetext{
*For collaboration list, see PoS(ICRC2019) 1177.

${ }^{\dagger}$ Speaker.
} 


\section{1. 'Secluded' dark matter and its signals at ANTARES}

The identification of the nature of Dark Matter (DM) constitutes a primary goal of modern physics. Several appealing theory extensions of the Standard Model (SM) feature particle DM candidates; among them WIMPs, with a mass at the electroweak scale, have motivated a tremendous experimental effort. This 30-years-long effort has not resulted in a discovery so far, pushing many WIMP DM models into uncomfortable regions of their parameter space. This situation is encouraging the exploration of other mass regimes for DM, in particular that of DM heavier than $O(10)$ $\mathrm{TeV}$ (for a pioneering theoretical study see e.g. the supersymmetric scenarios of [1]). This mass range is also of interest in light of the empty-handed searches for physics beyond the SM at the LHC, whose lesson could well be that the needed New Physics (NP) is relevant at energy scales larger than a few $\mathrm{TeV}$. It is then natural to expect that these NP sectors may host DM candidates with a similar mass.

High-energy neutrinos constitute one of the very few direct accesses to energy scales above a few TeV. This places the ANTARES telescope in a privileged position to test this relatively unexplored mass range for DM, via the search for neutrinos possibly coming from DM annihilations or decays. This position is reinforced by the favourable orientation of the Galactic Center, where most of the indirect signal from DM is expected to originate, with respect to the telescope. It appears therefore very well motivated to exploit ANTARES data to test models of DM heavier than a few TeV.

Among such models, as pointed out in [2] 'secluded DM' [3] constitute an ideal target for indirect detection. In these models the DM interacts sizeably with a mediator, itself interacting feebly with standard model particles. One can therefore have large indirect detection signals (because controlled by the DM-mediator interaction) with almost no signal in direct detection and collider experiments (because controlled by the small mediator-SM coupling). These models are particularly suited to describe heavy DM because

$\diamond$ [4] they allow to evade the so-called unitarity bound on the mass of thermal DM $m_{D M}<$ $O(100) \mathrm{TeV}[5,6]$, thanks to the late time entropy injection from decays of the mediator. $\mathrm{DM}$ masses of $100 \mathrm{TeV}$ and above become therefore allowed;

$\diamond$ [2] they overcome the technical difficulty of computing the final SM spectra, because the relevant energy scale is not the heavy DM mass (that for $m_{D M}>O(10) \mathrm{TeV}$ would demand a resummation of EW radiation) but rather the sub- $\mathrm{TeV}$ mediator mass, where the first order treatment of EW corrections [7] implemented in [8] is well under control.

The neutrino signal at ANTARES arises from the annihilation of DM pairs into two mediators, that then decay into neutrinos and/or SM particles that will produce neutrinos via decays and showering. The mediator lifetime is required to be shorter than $\mathrm{O}(0.1$ second) to respect limits from Big Bang Nucleosynthesis [9], so that it is instantaneous from the astrophysical point of view. The formation of positronium-like bound states of DM can sizeably contribute to the signal of interest for ANTARES, via the decay of the bound state into two or more mediators [10]. The DM annihilation cross section, for which sensitivities will be presented here, is then to be intended as 
an effective cross section taking into account also the bound state contribution (see e.g. [11, 12] for more details).

The energy spectra of the neutrinos per single DM annihilations are determined as follows: one first computes the energy spectra of neutrinos from the decay of a mediator at rest, using the tool [8], and then boosts them to the center of mass frame of the DM pair that annihilates, see [13] for more details. The resulting spectra are showed in Figure 1 for two benchmark values of the mediator mass. The 'primary' energy spectra above coincide with the spectra at the ANTARES location, as neutrinos of these energies propagate undisturbed in the Galaxy.
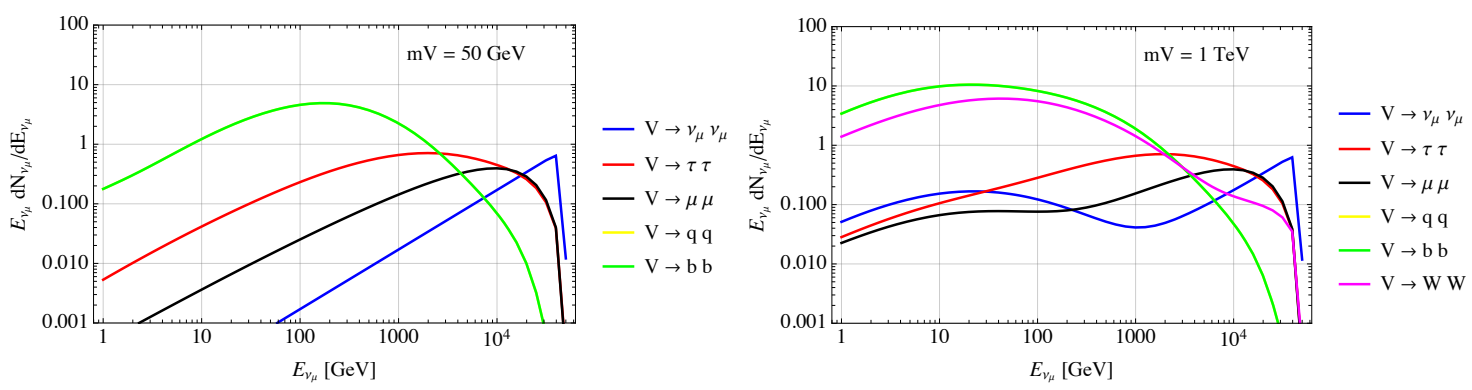

Figure 1: Energy distribution of the muon neutrinos plus antineutrinos at Earth location, per single annihilation of a DM pair into a two mediators. The mediator decays to the SM pair indicated in the legend, then all (anti)neutrino flavours coming from that specific pair are included and contribute via long-distance oscillations to the muon (anti)neutrinos at Earth location. The DM mass is fixed to $50 \mathrm{TeV} / \mathrm{c}^{2}$, the mediator mass to $50 \mathrm{GeV} / \mathrm{c}^{2}$ in the left-hand plot and to $1 \mathrm{TeV} / \mathrm{c}^{2}$ in the right-hand one.

\section{Analysis with the ANTARES neutrino telescope}

\subsection{Data Set}

To test heavy DM scenarios the sub- $\mathrm{TeV}$ to $200 \mathrm{TeV}$ mass region is investigated, a relatively high-energy regime compared with typical dark matter searches. The Galactic Centre is selected as a target not only for its large J-factor, but especially because for a source in the Southern Hemisphere, ANTARES (latitude $42^{\circ} 48^{\prime} \mathrm{N}$ ) can extend towards high energies with an increase of effective volume and in track reconstruction quality.

In this analysis the sensitivity to secluded dark matter is computed with 9 years of data taken with the ANTARES neutrino telescope [14] between May 2007 and December 2015, over an effective lifetime of 2101.6 days. The analysis is optimised on muon events from CC $v_{\mu}$ interactions, which result in the detector with the topology of a track, reconstructed with a good angular resolution of less than 1 degree.

A standard set of simulated data produced in ANTARES in correspondence of each data run is adapted to reproduce the secluded dark-matter energy spectra mentioned in section 1 through the use of weights reproducing the energy distribution $d N_{v} / d E_{v}$ of each DM annihilation channel. This search is optimised on right-ascension shuffled (blind) data, see [15] for more details.

\subsection{Method}

The kind of signal expected from a dark-matter excess is a cluster of events distributed around 
the position of the Galactic Centre with energy features given by the spectra shown in figure 1. These spectra are used to reproduce the distribution of signal variables $\mathscr{S}$, whilst background variable distributions $\mathscr{B}$ are obtained from blind data. $\mathscr{S}$ and $\mathscr{B}$ are normalised and used as probability density functions (PDFs) to build a likelihood function

$$
\log \mathscr{L}\left(n_{s}\right)=\sum_{i=1}^{N} \log \left[n_{s} \mathscr{S}\left(\psi_{i}, N_{H I T S}^{i}, q_{i}\right)+n_{b g} \mathscr{B}\left(\delta_{i}, N_{H I T S}^{i}, q_{i}\right)\right]-n_{b g}-n_{s}
$$

associated to a skymap with $n_{s}$ signal events injected over a background of $n_{b}=N_{t o t}-n_{s}$, being $N_{\text {tot }}=7637$ the total amount of selected muon tracks in our data sample. A set of $10^{4}$ toy skymaps is generated for each value of $n_{s}=1 \ldots 50$ events, distributed around the Galactic Centre according to the NFW profile [16] smeared with the detector resolution, defined as the angular difference between generated and reconstructed track from Monte Carlo simulation. The function $-\log \mathscr{L}$ is minimised with TMinuit [17] to yield the best-fit number of events $n_{s}^{*}$ found as belonging to the signal cluster of $n_{s}$. The significance of a cluster is established by the test statistics TS, function of the ratio between maximum likelihood and pure background likelihood

$$
T S=-\log \frac{\mathscr{L}\left(n_{s}^{*}\right)}{\mathscr{L}\left(n_{s}=0\right)} .
$$

As the number of events in each set of pseudo-experiments is subject to fluctuations following a Poisson distribution, a transformation through a Poisson function $\mathscr{P}$ is performed, returning the TS as a function of the Poissonian mean $\mu$

$$
P(T S(\mu))=P\left(T S\left(n_{s}^{*}\right)\right) \cdot \mathscr{P}\left(n_{s}, \mu\right) .
$$

To this, a 15\% systematics on the number of detected events is expected with ANTARES [18]. This effect is included folding the above equation with a Gaussian smearing of $15 \%$ width.

The distributions $P(T S(\mu))$ is compared with the background median $\overline{T S}_{0}$ to obtain the $90 \%$ confidence level sensitivity on the number of cluster events, representing the minimum number of events $\mu_{90}$ that this search would be able to discriminate from the pure background case committing a fake negative error with a $10 \%$ rate or less. In agreement with Neyman's prescription [19], upper limits will be set equal to the sensitivity in case a value smaller than the background median is observed in the data.

To convert the number of events $\mu_{90}$ into an integral flux of neutrino events in the detector, we define the acceptance as integrated effective area modulated through the secluded spectra [2] here indicated as $d N_{V} / d E_{v}$, for each fixed mediator mass $m_{V}$ and dark-matter candidate mass $M$ :

$$
\mathscr{A}\left(M, m_{V}\right)=\int_{0}^{M} d E_{v} A_{e f f}^{v}\left(E_{v}\right) \frac{d N_{v}\left(E_{v}, m_{V}\right)}{d E_{v}}+\int_{0}^{M} d E_{\bar{v}} A_{e f f}^{\bar{v}}\left(E_{\bar{v}}\right) \frac{d N_{\bar{v}}\left(E_{\bar{v}}, m_{V}\right)}{d E_{\bar{v}}}
$$

where neutrino and anti-neutrino events are indistinguishably seen with ANTARES, so our measurement includes a sum of both. The flux of measured events is then

$$
\Phi(M)=\int_{0}^{M} d E_{v, \bar{v}} \frac{d \Phi\left(E_{v, \bar{v}}\right)}{d E_{v, \bar{v}}}=\frac{\mu_{90}}{\mathscr{A}(M) \cdot t}
$$

with $t$ being the effective lifetime of the analysis. Integrated acceptances are shown in figure 2 for two values of the mediator masses $m_{V}$. 

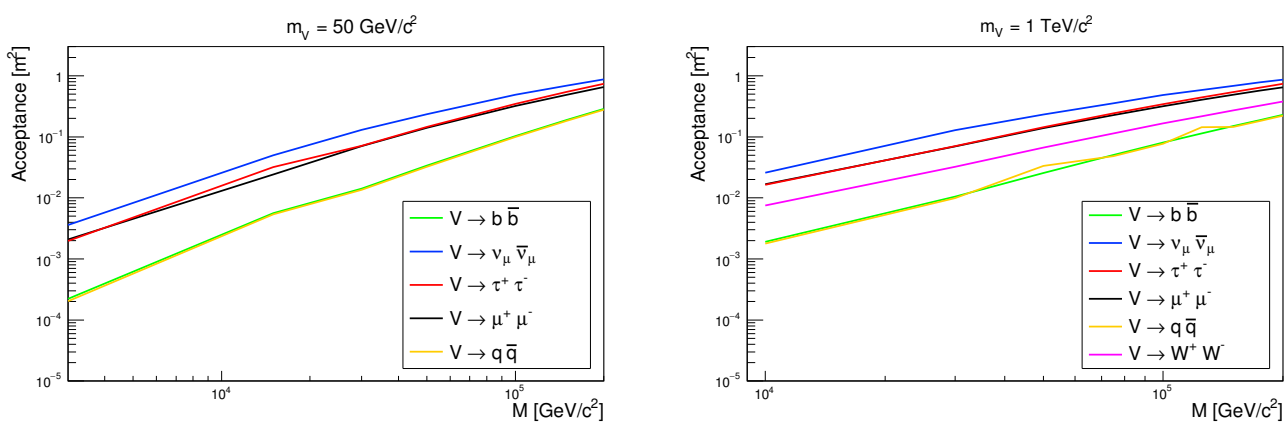

Figure 2: Integrated acceptances of the ANTARES detectors to secluded spectra, relative to a mediator with mass $m_{V}=50 \mathrm{GeV} / \mathrm{c}^{2}$ (left) and $m_{V}=1 \mathrm{TeV} / \mathrm{c}^{2}$ (right).

\section{Results and discussion}

For each value of $m_{V}=\{10,20,50,250,1000\} \mathrm{GeV} / \mathrm{c}^{2}$ we obtain sensitivities on the WIMP pair annihilation cross-sections from the relation

$$
\frac{d \Phi\left(E_{v}\right)}{d E_{v}}=\frac{1}{4 \pi M^{2}} \frac{\langle\sigma v\rangle}{2} \frac{d N\left(E_{v}\right)}{d E_{v}} J
$$

with the Galactic Centre morphology described by the J-Factor $J=\int d \Omega \int \rho^{2} d s$ with NFW model for $\rho(s)$ [16]. We use an integrated $J$-factor of $1.24 \cdot 10^{-23} \mathrm{GeV}^{2} \mathrm{~cm}^{-5}$ over an angle of $30^{\circ}$. Sensitivities obtained on the effective thermally averaged cross section for dark-matter pair annihilation into a mediator pair, $\langle\sigma v\rangle$, are shown in figure 3 for two reference mediator masses $m_{V}=50$ $\mathrm{GeV} / \mathrm{c}^{2}$ and $1 \mathrm{TeV} / \mathrm{c}^{2}$.
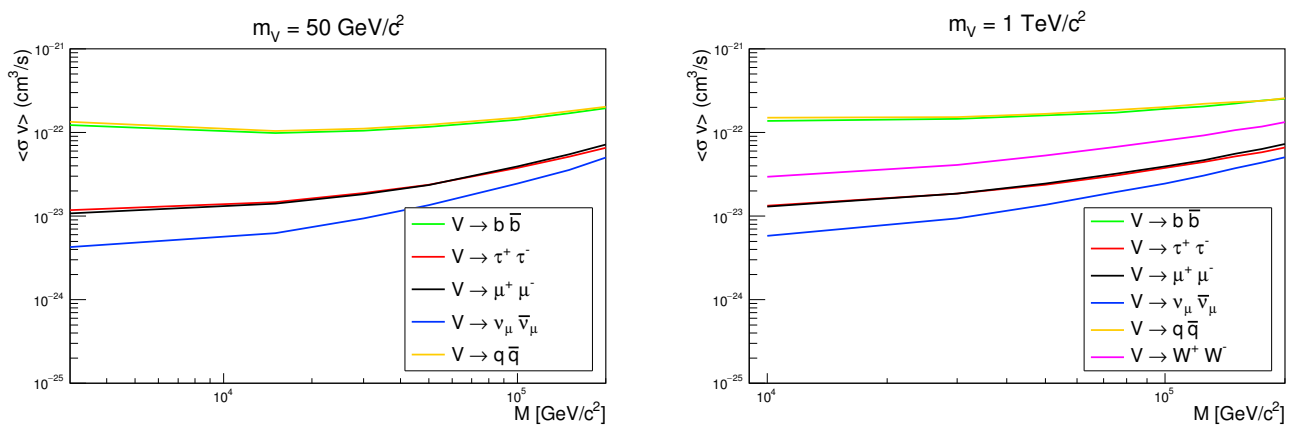

Figure 3: Sensitivities reached with 9 years of ANTARES data on the effective cross-section for DM pairannihilation into a mediator pair, where the label in the figure indicates the decay channel of a single mediator. The mediator mass is $m_{V}=50 \mathrm{GeV} / \mathrm{c}^{2}$ (left) and $m_{V}=1 \mathrm{TeV} / \mathrm{c}^{2}$ (right)

\section{Conclusions}

With this analysis we have examined the possibilities for ANTARES to test new dark-matter scenarios outside the typical parameter space of WIMP searches [20], focusing on models of secluded DM with a mass as high as $200 \mathrm{TeV}$. Given the favourable position of ANTARES with 
respect to Galactic Centre observations, this detector has good possibilities to extend standard dark-matter analyses towards high energies. The resulting sensitivities on the effective DM annihilation cross section are shown in figure 3; they are encouraging as they show that ANTARES could start testing the parameter space of specific secluded DM models [2].

Further improvements of this work are under study, like the introduction of a selection based on dedicated spectral features, with a possible inclusion of $v_{e}$ events which measured with ANTARES which are reconstructed with more accurate energy resolution. Testing secluded dark-matter predictions at high energies is as well an interesting target for KM3NeT/ARCA, the high-energy unit of KM3NeT [21], currently under construction in the Mediterranean Sea and whose science case is oriented to neutrino astrophysics.

\section{Acknowledgements}

We gratefully acknowledge the financial support of the Ministerio de Ciencia, Innovación y Universidades: Programa Estatal de Generación de Conocmiento, ref. PGC2018-096663-B-C41 (MCIU/FEDER) and Severo Ochoa Centre of Excellence (MCIU), Spain.

\section{References}

[1] S. Dimopoulos, G. F. Giudice, and A. Pomarol, Phys. Lett. B389 (1996) 37-42.

[2] M. Cirelli, Y. Gouttenoire, K. Petraki, and F. Sala, Journal of Cosmology and Astro-Particle Physics 2019 (Feb, 2019) 014.

[3] M. Pospelov, A. Ritz, and M. B. Voloshin, Phys. Lett. B662 (2008) 53-61.

[4] A. Berlin, D. Hooper, and G. Krnjaic, Phys. Lett. B760 (2016) 106-111.

[5] K. Griest and M. Kamionkowski, Phys. Rev. Lett. 64 (1990) 615.

[6] J. Smirnov and J. F. Beacom, arXiv:1904.11503.

[7] P. Ciafaloni, D. Comelli, A. Riotto, F. Sala, A. Strumia, and A. Urbano, JCAP 1103 (2011) 019.

[8] M. Cirelli, G. Corcella, A. Hektor, G. Hutsi, M. Kadastik, P. Panci, M. Raidal, F. Sala, and A. Strumia, JCAP 1103 (2011) 051. [Erratum: JCAP1210,E01(2012)].

[9] K. Jedamzik, Phys. Rev. D74 (2006) 103509.

[10] M. Pospelov and A. Ritz, Phys. Lett. B671 (2009) 391-397.

[11] K. Petraki, M. Postma, and J. de Vries, JHEP 04 (2017) 077.

[12] M. Cirelli, P. Panci, K. Petraki, F. Sala, and M. Taoso, JCAP 1705 (2017) 036.

[13] G. Elor, N. L. Rodd, T. R. Slatyer, and W. Xue, JCAP 1606 (2016) 024.

[14] M. Ageron, J. A. Aguilar, I. Al Samarai, A. Albert, F. Ameli, M. André, M. Anghinolfi, G. Anton, S. Anvar, and M. Ardid, Nuclear Instruments and Methods in Physics Research A 656 (Nov, 2011) $11-38$.

[15] M. Ageron, J. A. Aguilar, I. Al Samarai, A. Albert, F. Ameli, M. André, M. Anghinolfi, G. Anton, S. Anvar, and M. Ardid, These proceedings.

[16] J. F. Navarro, C. S. Frenk, and S. D. M. White, Astrophys. J. 462 (1996) 563. 
[17] R. Brun and F. Rademakers, Nuclear Instruments and Methods in Physics Research A 389 (Feb, 1997) 81-86.

[18] A. Albert, M. André, M. Anghinolfi, G. Anton, M. Ardid, J. J. Aubert, T. Avgitas, B. Baret, J. Barrios-Martí, and S. Basa, 96 (Oct, 2017) 082001.

[19] J. Neyman, Lectures and Conferences on Mathematical Statistics. 1938.

[20] A. Albert, M. André, M. Anghinolfi, G. Anton, M. Ardid, J. J. Aubert, T. Avgitas, B. Baret, J. Barrios-Martí, and S. Basa, Physics Letters B 769 (Jun, 2017) 249-254. [Erratum: Phys. Lett.B(2019)].

[21] S. Adrián-Martínez, M. Ageron, F. Aharonian, S. Aiello, A. Albert, F. Ameli, E. Anassontzis, M. Andre, G. Androulakis, and M. Anghinolfi, Journal of Physics G Nuclear Physics 43 (Aug, 2016) 084001. 